\title{
ALMA observations of Molecules in Supernova 1987A
}

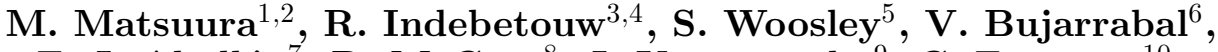
F. J. Abellán ${ }^{7}$, R. McCray ${ }^{8}$, J. Kamenetzky ${ }^{9}$, C. Fransson ${ }^{10}$, M. J. Barlow ${ }^{2}$, H. L. Gomez ${ }^{1}$, P. $\operatorname{Cigan}^{1}$, I De Looze ${ }^{2}$, J. Spyromilio ${ }^{11}$, L. Staveley-Smith ${ }^{12,13}$, G. Zanardo ${ }^{12}$, P. Roche ${ }^{14}$, J. Larsson ${ }^{15}$, S. Viti ${ }^{2}$, J. Th. van Loon ${ }^{16}$, J. C. Wheeler ${ }^{17}$, M. Baes ${ }^{18}$, R. Chevalier $^{3}$, P. Lundqvist ${ }^{10}$, J. M. Marcaide ${ }^{7}$, E. Dwek ${ }^{19}$, M. Meixner ${ }^{20,21}$, C.-Y. $\mathbf{N g}^{22}$, G. Sonneborn ${ }^{19}$ and J. Yates $^{2}$

${ }^{1}$ School of Physics and Astronomy, Cardiff University, Queen's Buildings, The Parade, Cardiff, CF24 3AA, UK

${ }^{2}$ Department of Physics and Astronomy, University College London, Gower Street, London WC1E 6BT, UK

${ }^{3}$ Department of Astronomy, University of Virginia, P.O. Box 400325, Charlottesville, VA 22904-4325, USA

${ }^{4}$ National Radio Astronomy Observatory, 520 Edgemont Rd, Charlottesville, VA 22903, USA

${ }^{5}$ Department of Astronomy and Astrophysics, University of California, Santa Cruz, CA 95064, USA

${ }^{6}$ Observatorio Astronómico Nacional (OAN-IGN), Apartado 112, E-28803 Alcalá de Henares, Spain

${ }^{7}$ Universidad de Valencia, C/Dr. Moliner 50, E-46100 Burjassot, Spain

${ }^{8}$ Department of Astronomy, University of California, Berkeley, CA 94720-3411, USA

${ }^{9}$ Westminster College, Department of Physics, 1840 South 1300 East, Salt Lake City, UT 84105, USA

${ }^{10}$ Department of Astronomy, The Oskar Klein Centre, Stockholm University, Alba Nova University Centre, SE-106 91 Stockholm, Sweden

${ }^{11}$ European Southern Observatory (ESO), Karl-Schwarzschild-Strasse 2, 85748 Garching, Germany

${ }^{12}$ International Centre for Radio Astronomy Research (ICRAR), The University of Western Australia, Crawley, WA 6009, Australia

${ }^{13}$ ARC Centre of Excellence for All-Sky Astrophysics (CAASTRO), Australia

${ }^{14}$ Department of Physics, University of Oxford, Oxford OX1 3RH, UK

${ }^{15} \mathrm{KTH}$, Department of Physics, and the Oskar Klein Centre, AlbaNova, SE-106 91 Stockholm, Sweden

${ }^{16}$ School of Physical and Geographical Sciences, Lennard-Jones Laboratories, Keele University, Staffordshire ST5 5BG, UK

${ }^{17}$ Department of Astronomy, University of Texas, Austin, TX 78712-0259, USA

${ }^{18}$ Sterrenkundig Observatorium, Universiteit Gent, Krijgslaan 281 S9, B-9000 Gent, Belgium

${ }^{19}$ Observational Cosmology Lab, Code 665, NASA Goddard Space Flight Center, Greenbelt, MD 20771, USA

${ }^{20}$ Space Telescope Science Institute, 3700 San Martin Dr., Baltimore, MD 21218, USA

${ }^{21}$ Department of Physics and Astronomy, The Johns Hopkins University, 366 Bloomberg Center, 3400 N. Charles Street, Baltimore, MD 21218, USA

${ }^{22}$ Department of Physics, The University of Hong Kong, Pokfulam Road, Hong Kong, China

Abstract. Supernova (SN) 1987A has provided a unique opportunity to study how SN ejecta evolve in 30 years time scale. We report our ALMA spectral observations of SN 1987A, taken in 2014, 2015 and 2016, with detections of $\mathrm{CO},{ }^{28} \mathrm{SiO}, \mathrm{HCO}^{+}$and $\mathrm{SO}$, with weaker lines of ${ }^{29} \mathrm{SiO}$.

We find a dip in the $\mathrm{SiO}$ line profiles, suggesting that the ejecta morphology is likely elongated. The difference of the $\mathrm{CO}$ and $\mathrm{SiO}$ line profiles is consistent with hydrodynamic simulations, which 
show that Rayleigh-Taylor instabilities causes mixing of gas, with heavier elements much more disturbed, making more elongated structure.

Using ${ }^{28} \mathrm{SiO}$ and its isotopologues, $\mathrm{Si}$ isotope ratios were estimated for the first time in SN 1987A. The estimated ratios appear to be consistent with theoretical predictions of inefficient formation of neutron rich atoms at lower metallicity, such as observed in the Large Magellanic Cloud (about half a solar metallicity).

The deduced large $\mathrm{HCO}^{+}$mass and small SiS mass, which are inconsistent to the predictions of chemical model, might be explained by some mixing of elements immediately after the explosion. The mixing might have made some hydrogen from the envelope to sink into carbon and oxygenrich zone during early days after the explosion, enabling the formation of a substantial mass of $\mathrm{HCO}^{+}$. Oxygen atoms may penetrate into silicon and sulphur zone, suppressing formation of SiS.

Our ALMA observations open up a new window to investigate chemistry, dynamics and explosive-nucleosynthesis in supernovae.

Keywords. supernovae: individual:Supernova 1987A — ISM: supernova remnants - ISM: molecules — ISM: abundances — radio lines: ISM

\section{Introduction}

Core-collapse supernovae ( $\mathrm{SNe}$ ) are important source of metal content in galaxies. Inside the stellar interior, metals are synthesised and newly synthesised elements are ejected from SNe, that enrich the interstellar medium of galaxies. So far, most abundance measurements of SN remnants have focused on detecting lines at X-ray, UV and optical wavelengths. Constraints obtained from these short wavelength spectra are limited to the atomic lines of the main isotope only. ALMA detection of molecules in SN 1987A provide a different angle in abundance measurements, by investigating isotope abundances in SNe, using molecular lines. Further, ALMA can investigate molecular chemistry occurring in early phase of evolution in SN 1987A. Here, we report our spectroscopic survey of SN 1987A from Matsuura et al. 2017.

\section{Observations and data reduction}

ALMA obtained spectra covering a continuous spectral range from 210 to $300 \mathrm{GHz}$, in August and September 2014. Additionally, $\mathrm{CO} J=3-2$ and $357 \mathrm{GHz} \mathrm{HCO}^{+} \mathrm{J}=4-3$ was obtained on 2015 July 25 th A series of slightly overlapping $\sim 2000 \mathrm{~km} \mathrm{~s}^{-1}$ wide segments were observed in sequence; whereas a typical SN 1987A ejecta line width is $2000 \mathrm{~km} \mathrm{~s}^{-1}$ (Kamenetzky et al. 2013), so that one emission line can fill one ALMA spectrum segment.

\section{ALMA Spectrum}

In Figure 1 we show the observed spectrum of SN 1987A's ejecta, showing a variety of broad molecular lines. The strongest molecular emissions are attributed to $\mathrm{SiO}$ and $\mathrm{CO}$. The emission at $267 \mathrm{GHz}$ is associated with $\mathrm{HCO}^{+} J=3-2$, with some possible contamination from $\mathrm{SO}_{2}$. Other weak features are due to $\mathrm{SO}$ and ${ }^{29} \mathrm{SiO}$.

It has been thought that supernovae are molecule-poor environments, because hydrogen, the key element for chemical reaction, is expected to be rather deficient in SN ejecta, and because $\mathrm{He}^{+}$destroys molecules formed within it (Lepp et al. 1990, Rawlings \& Williams, Sarangi \& Cherchneff 2013). For young (<10 years old) SNe, CO and SiO had been the only molecules firmly detected (Matsuura 2017), with a recent report of 

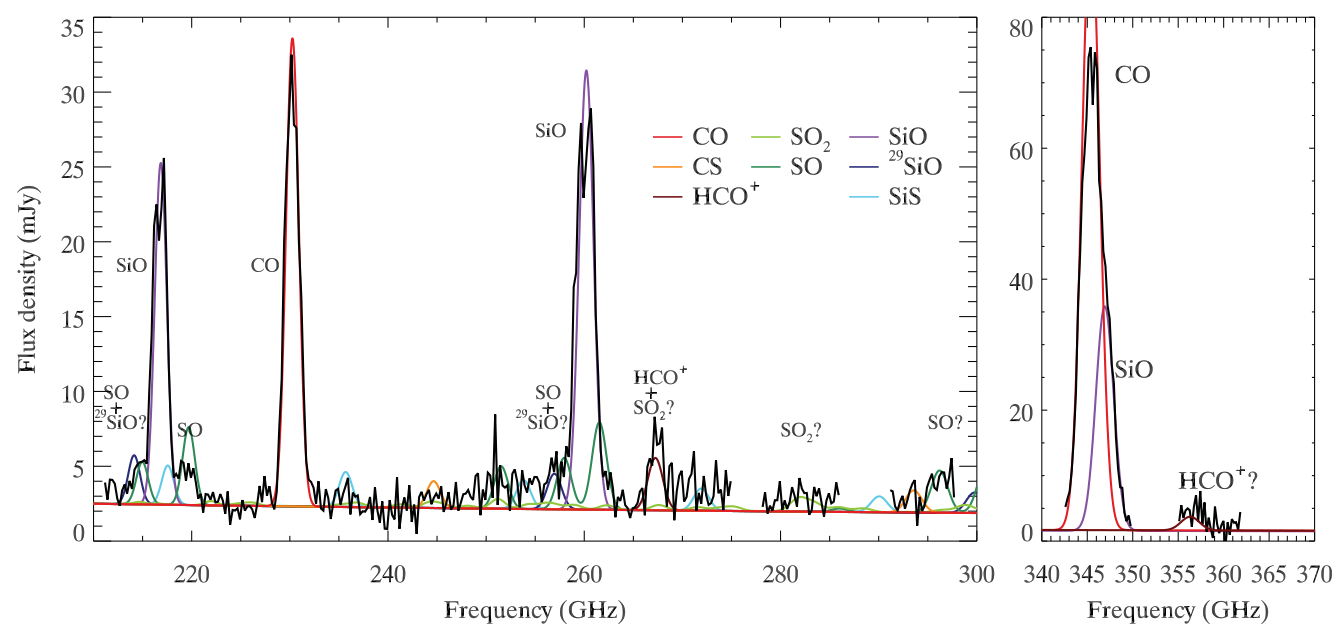

Figure 1. The ALMA $210-300$ and 340-360 GHz spectra of SN 1987A's ejecta (black). Model molecular spectra with a $2000 \mathrm{~km} \mathrm{~s}^{-1}$ with FWHM Gaussian line profile and the excitation temperature of $40 \mathrm{~K}$, are plotted in colour, guiding molecular identifications.

detecting near-infrared $\mathrm{H}_{2}$ lines from SN 1987A (Fransson et al. 2016). Now from our spectral line survey with ALMA, we have discovered $\mathrm{SO}$ and $\mathrm{HCO}^{+}$in the ejecta of SN 1987A. These detections add to the already detected molecules at millimetre wavelengths, $\mathrm{CO}$ and $\mathrm{SiO}$, and likely ${ }^{29} \mathrm{SiO}$ (Kamenetzky et al. 2013). The ejecta of young $\mathrm{SN}$ remnants may represent at a much more molecule-rich environment than had been thought before.

\section{Discussion}

\subsection{1. $\mathrm{HCO}^{+}$as a tracer of dense gas and possible requirement of mixing}

The detection of $\mathrm{HCO}^{+}$in SN 1987A was surprising. Chemical models by Sarangi \& Cherchneff (2013) did not consider $\mathrm{HCO}^{+}$formation, while Rawlings \& Williams (1990) did consider it but predicted very small $\mathrm{HCO}^{+}$mass.

Forming $\mathrm{HCO}^{+}$requires $\mathrm{H}_{2}$, which is abundant only in the hydrogen envelope. Forming $\mathrm{HCO}^{+}$also requires $\mathrm{H}_{2}$ in $\mathrm{CO}$ gas, as following reaction $\mathrm{H}_{3}^{+}+\mathrm{CO} \rightarrow \mathrm{H}_{2}+\mathrm{HCO}^{+}$, and $\mathrm{H}_{2}^{+}+\mathrm{H}_{2} \rightarrow \mathrm{H}_{3}^{+}+\mathrm{H}$ (Williams \& Viti 2014). There is a small fraction of $\mathrm{C}$ and $\mathrm{O}$ in the hydrogen envelope, because $\mathrm{C}$ and $\mathrm{O}$ were incorporated into the star at the time of star formation. However, the masses of intrinsic $\mathrm{C}$ and $\mathrm{O}$ are insufficient to account for the $\mathrm{CO}$ mass needed to generate substantial $\mathrm{HCO}^{+}$mass.

Forming a large mass of $\mathrm{HCO}^{+}$may have been enabled by macroscopic mixing in the ejecta early phases after the SN explosion, and that process might mix $\mathrm{C}$ and $\mathrm{O}$ in the stellar core and $\mathrm{H}$ in hydrogen envelope. A classic picture of stellar nucleosynthesis is that the progenitor undergoes a sequence of nuclear reactions in the stellar interiors, making onion-type shells composed of multiple zones with discrete elemental abundances. Figure 2 (a) shows the radial distributions of element mass without mixing. This figure is based on explosive nucleosynthesis models for SN 1987A (Sukhbold et al. 2016, Woosley 1988 and Woosley et al. 1997). Figure 2 (b) illustrate the effect of mixing; if there is some fraction of hydrogen mixed with carbon and oxygen. Instead of mixing elements completely, mixing might occur locally at the interfaces of the He and He-envelopes and the $\mathrm{He}$ and $\mathrm{C}+\mathrm{O}$ zone, due to Rayleigh-Taylor instabilities (Müller et al. 1991, 
(a) Unmixed

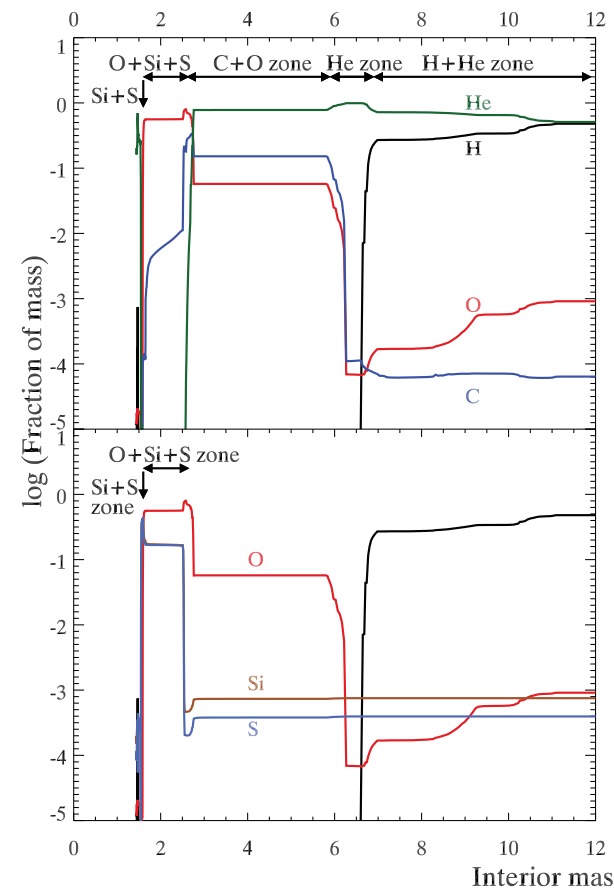

(b) Mixed

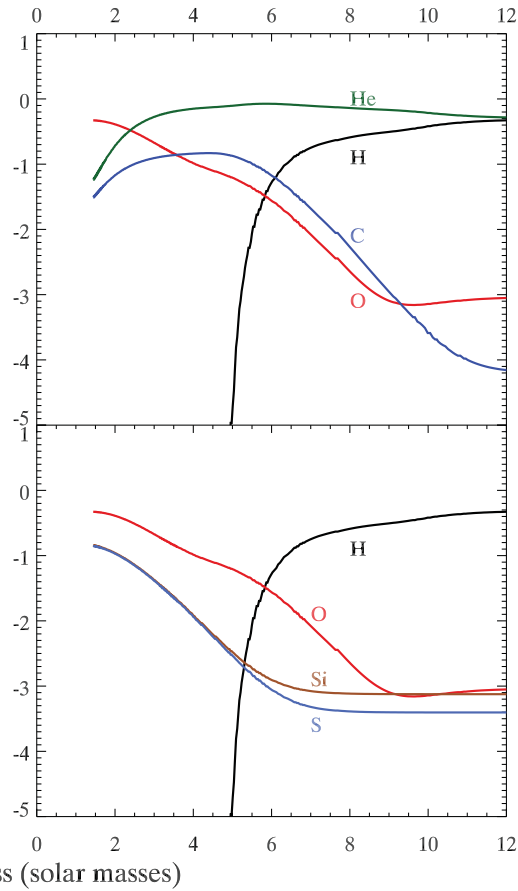

Figure 2. The modelled fractional abundance of atoms after SN explosion. The model is an $18 M_{\odot}$ star with one third of the solar metallicity based on the model for Sk-69 9202 (Sukhbold et al. 2016), but involving more extensive nuclear reaction network. Panel (a) shows SN 1987A model without mixing of nuclear burning zones. Panel (b) illustrates the effect of mixing, with the model with artificial mixing at $t=10^{7} \mathrm{~s}$. Having mixing triggered by the explosion can make elements from different nuclear burning zones mixed to some degree, though the scale of mixing is undetermined.

Wongwathanarat et al. 2015). The presence of a such large scale mixing at early times could have later resulted in a substantial mass of $\mathrm{HCO}^{+}$.

\subsubsection{SiO and SiS chemistry}

Chemical models predict that the majority of $\mathrm{Si}$ is locked up in silicate dust grains at day 1500, and not so much $\mathrm{SiO}$ is present (Sarangi \& Cherchneff 2013, Sarangi \& Cherchneff 2015). Our measured $\mathrm{SiO}$ mass is consistent with this hypothesis qualitatively, but not quantitatively. Our estimated $\mathrm{SiO}$ mass is $4 \times 10^{-5}-2 \times 10^{-3} M_{\odot}$ which is a factor of 16-800 larger than the theoretically predicted $\mathrm{SiO}$ mass of $2.5 \times 10^{-6} M_{\odot}$ (Sarangi \& Cherchneff 2013). Nevertheless, $\mathrm{Si}$ atoms in $\mathrm{SiO}$ is only $3 \times 10^{-5}-1 \times 10^{-3} M_{\odot}$ which corresponds to less than $10 \%$ of Si mass synthesised in the SN. Indeed, a large fraction of Si could be in silicate dust

Chemical models (Sarangi \& Cherchneff 2013) predict that the majority of Si is in SiS rather than $\mathrm{SiO}$, and the predicted $\mathrm{SiS}$ mass is $4.4 \times 10^{-2} M_{\odot}$. However, we find only small mass of $\operatorname{SiS}\left(<6 \times 10^{-5} M_{\odot}\right)$.

We argue that a large $\mathrm{SiO}$ abundance and a small $\mathrm{SiS}$ abundance suggests the presence of some degree of mixing. Figure 2 (b) shows an example of a mixed case. At a time of $10^{7} \mathrm{~s}(\sim 116$ days $)$ after the explosion, $\mathrm{Si}$ and $\mathrm{S}$ atoms are mixed with $\mathrm{O}$ atoms. The mixing enables one to form $\mathrm{SiO}$ more efficiently from co-existing $\mathrm{Si}$ and $\mathrm{O}$ atoms. 


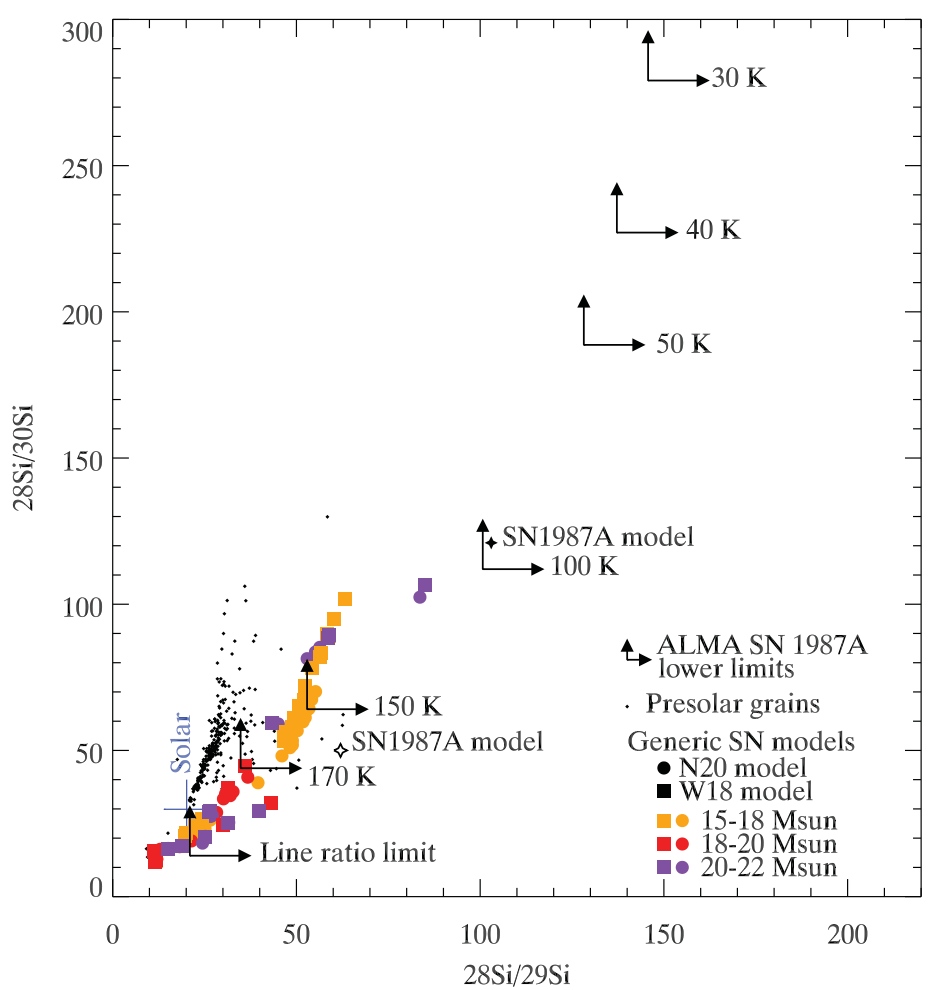

Figure 3. The Si isotope ratios. ALMA lower limits for SN 1987A are plotted with arrows, as a function of the assumed kinetic temperature of the molecule. The measured lower limits are compared with explosive nucleosynthesis models, including SN 1987A specific models (filled star: based on Woosley et al. 1988; open star: based on Nomoto et al. 2013). Generic solar abundance models are from Sukhbold et al. (2016), as a function of zero-age main sequence mass.

\subsection{Isotope ratios}

Our ALMA spectrum can cover the lines of $\mathrm{SiO}$ and $\mathrm{CO}$ isotopologues. That can be used to estimate lower limits for the isotope ratios of SN 1987A. Together with the Crab Nebula measurements of Ar isotope ratios (Barlow et al. 2013), SN 1987A is one of the first to estimate the isotope ratios for core-collapse SNe (Kamenetzky et al. 2013).

Fig. 3 shows our ALMA lower limits for ${ }^{28} \mathrm{Si} /{ }^{29} \mathrm{Si}$ and ${ }^{28} \mathrm{Si} /{ }^{30} \mathrm{Si}$, as a function of the assumed RADEX kinetic temperature. These ALMA lower limits are compared with theoretically predicted ratios from explosive nucleosynthesis models based on Sukhbold et al. (2016).

The ratio of ${ }^{30} \mathrm{Si} /{ }^{28} \mathrm{Si}$ depends on the neutron excess, which depends on the initial metallicity of the star (Woosley 1988). Lower metallicity gives a lower neutron excess, resulting in a smaller production of neutron-rich isotopes. Because ${ }^{30} \mathrm{Si}$ requires more neutrons than ${ }^{29} \mathrm{Si}$, and even more than ${ }^{28} \mathrm{Si},{ }^{28} \mathrm{Si} /{ }^{30} \mathrm{Si}$ becomes even larger than ${ }^{28} \mathrm{Si} /{ }^{29} \mathrm{Si}$ at lower metallicity, compared to the solar metallicity.

\section{Acknowledgments}

This paper makes use of the following ALMA data: ADS/JAO.ALMA\#2013.1.00280.S, ADS/JAO.ALMA\#2013.1.00063.S and ADS/JAO.ALMA\#2012.1.00075.S. ALMA is a partnership of ESO (representing its member states), NSF (USA) and NINS (Japan), to- 
gether with NRC (Canada), NSC and ASIAA (Taiwan), and KASI (Republic of Korea), in cooperation with the Republic of Chile. The Joint ALMA Observatory is operated by ESO, AUI/NRAO and NAOJ. MM and MJB acknowledge support from UK STFC grant (ST/J001511/1), and MM is further supported by an STFC Ernest Rutherford fellowship (ST/L003597/1). MJB acknowledges support from European Research Council (ERC) Advanced Grant SNDUST 694520. HLG acknowledges support from the European Research Council (ERC) in the form of Consolidator Grant CosmicDust (ERC-2014-CoG647939). SW acknowledges support from supported by NASA grant (NNX14AH34G).

\section{References}

Barlow, M. J., Swinyard, B. M., Owen, P. J., Cernicharo, J., Gomez, H. L., Ivison, R. J., Krause, O., Lim, T. L., Matsuura, M., Miller, S., Olofsson, G., \& Polehampton, E. T., 2013, Science, 342,1343

Fransson, C., Larsson, J., Spyromilio, J., Leibundgut, B., McCray, R. A., \& Jerkstrand, A., 2016, ApJ, 821, L5

Lepp, S., Dalgarno, A., \& McCray, R. A., 1990, ApJ, 358, 262

Kamenetzky, J., McCray, R. A., Indebetouw, R., et al. 2013, ApJ, 773, L34

Matsuura, M., Indebetouw, R., Woosley, S. et al., 2017, MNRAS, in press (arXiv/17)04.02324

Matsuura, M., "Handbook of Supernovae", Springer International Publishing, Ed. Athem W. Alsabti \& Paul Murdin

Müller E., Fryxell, B., \& Arnett, W. D., 1991, A\&A, 251, 505

Nomoto, K., Kobayashi, C., \& Tominaga, N., 2013, ARA\&A, 51, 457

Rawlings, J. M. C. \& Williams, D. A., 1990, MNRAS, 246, 208

Sarangi, A. \& Cherchneff, I., 2013, ApJ, 776, 107

Sarangi, A. \& Cherchneff, I., 2015, A\& A, 575, A95

Sukhbold, T., Ertl, T., Woosley, S. E., Brown, J. M., \& Janka, H. T., 2016, ApJ, 821, 38

Williams, D. A. \& Viti Serena, S., 2014, Observational Molecular Astronomy. Cambridge University Press

Wongwathanarat, A., Muller, E., \& Janka, H. T. T., 2015, A\& A, 577, A48

Woosley, S. E., 1988, ApJ, 330, 218

Woosley, S. E., Pinto, P. A., \& Weaver, T. A., 1988, Australian Academy of Science, 7, 355

Woosley, S. E., Heger, A., Weaver, T. A., \& Langer, N., 1997, in Phillips M., Suntzeff N., eds, SN 1987A: Ten years after SN 1987A - Presupernova Evolution and the Progenitor Star. PASPC, pp (astro-ph/9705146) 